\title{
Effect of Lime and FYM on the Herb Yield of Mint (Mentha arvensis) in an Acid Soil Odisha
}

\author{
Sunita Kumari ${ }^{1}$, P.K. Das ${ }^{1}$, Abhay Kumar $^{2 *}$ and P.R. Oraon ${ }^{2}$ \\ ${ }^{1}$ Department of forest product and utilization, College of Forestry, OUAT, \\ Bhubaneswar, Odisha-751003, India \\ ${ }^{2}$ Faculty of forestry, Birsa Agricultural University, Kanke, Ranchi, Jharkhand-834006, India \\ *Corresponding author
}

\section{A B S T R A C T}

\section{Keywords}

Mint, Lime, FYM, Medicinal plant.

Article Info

Accepted:

24 September 2017

Available Online:

10 November 2017
A field experiment was conducted in the nursery of the College of Forestry, OUAT, Bhubaneswar to study the effect of lime and FYM applied alone and in different combinations on the yield of Mentha arvensis. The experiment was designed in Factorial RBD with nine treatments and three replications. The treatments consist of liming @ 0, 0.3 and 0.5 LR with FYM @ 0, 2.5 and 5.0 ton/ha in different combinations. Results of the experiment revealed that application of lime and FYM significantly increased the plant height; root, leaf and stem weights; leaf, herbage and oil yields. Maximum leaf, herbage and oil yields of $110 \mathrm{q} / \mathrm{ha}, 318 \mathrm{q} / \mathrm{ha}$ and $44 \mathrm{~kg} / \mathrm{ha}$, respectively (after 120 days of planting) were obtained with application of lime @0.3LR along with FYM @5 ton/ha. These yields were 157, 122 and 487\% higher, respectively, than these in control (0lime +0 FYM). Maximum leaf, herbage and oil yield after180 days of planting were the highest $(84 \mathrm{q} / \mathrm{ha}, 330 \mathrm{q} / \mathrm{ha}$ and $30 \mathrm{~kg} / \mathrm{ha}$, respectively) when lime was applied @ 0.5 LR along with FYM @ 5.0 ton/ha. These yields were 123, 120 and $400 \%$ higher, respectively, than those in the control. Application of lime had no effect in increasing the oil content, whereas FYM had a significant effect in increasing the oil content in $M$. arvensis.

\section{Introduction}

Mentha arvensis L., popularly known as Japanese mint is an aromatic and medicinal plant that belongs to the Lamiaceae family (Lorenziand Matos, 2002). It is world's third most valuable flavouring agent (Fenarolics, 1971). Different species of the family Lamiaceae have been cultivated in different parts of the world with major producers of mint are India, China, Vietnam and Brazil (Chand et al., 2004; Clark, 1998; Kumar et al., 2000; Lawrence, 2007; Singh and Saini, 2008). Mentha species reproduce both by reproductive and vegetative means. The area under menthol mint is mainly confined to central parts of Indo-Gangatic plains encompassing the northern states of Punjab, Haryana, Uttar Pradesh, Biharandtarai belt of Uttarakhand (Kumar et al., 2001). The menthol mint (Mentha arvensis L.), a potent source of menthol oil has occupied a prime 
position in Indian agriculture due to its number of industrial applications. It has emerged as a competent cash crop in north Indian plains in view of high market price for its essential mint oil. India is a leading producer of mint oil, contributing approximately $85 \%$ of the total world production (Anwar et al., 2010). In India, it is cultivated on 1.75 lakh ha area with a production of 55000 tonnes (www.businessstandard.com). Presently about $75 \%$ of the world requirement of menthol crystallized from mint oil is met by India. India exports approximately 25,000 tonnes all forms of menthol oil including crystals. The domestic consumption of India is about $40 \%$ of global consumption. Japanese or menthol mint (Mentha arvensis L.) is a succulent, multi-cut perennial crop containing $75-85 \%$ menthol. Mint oil has wide applications in pharmaceutical, agro-chemicals and flavoring industries (Tassou et al., 2004).

\section{Materials and Methods}

The field experiment was conducted in the nursery of the College of Forestry, Orissa University of Agriculture and Technology, Bhubaneswar. The soil of the experimental site was sandy loam with $17.6 \%$ clay. The soil was strongly acidic having the $\mathrm{pH}$ of 5.4. Salt $\mathrm{pH}$ with $0.01 \mathrm{CaCl}_{2}$ was 5.0 positive difference between $\mathrm{pH}$ and salt $\mathrm{pH}$ indicated that the soil was net negatively charged. Organic carbon content in the soil was low $(0.46 \%)$. Available $\mathrm{N}$ (alkaline $\mathrm{KMnO}_{4}$ method), P (Brays' I method), $\mathrm{K}$ ( $\left.\mathrm{NH}_{4} \mathrm{OAc}\right)$ were low $(108,23.5,14.6 \mathrm{~kg} / \mathrm{ha}$ respectively. Exchangeable $\mathrm{Ca}^{2+}+\mathrm{Mg}^{2+}$ content in the soil was $1.4 \mathrm{cmol}(\mathrm{p}+) \mathrm{kg}^{-1}$. Lime requirement of the soil to the $\mathrm{pH} 6.5$ determined by modified woodruff buffer method, was $2000 \mathrm{~kg} / \mathrm{ha}$ The experimental design was factorial RBD with nine treatment and three replications $\left(\mathrm{T}_{1}=0 \mathrm{LR}+0 \mathrm{FYM}, \mathrm{T}_{2}=0 \mathrm{LR}+\mathrm{FYM} @ 2.5 \mathrm{t} / \mathrm{ha}, \mathrm{T}_{3}=\right.$ 0LR + FYM@5t/ha, T $4=0.3 \mathrm{LR}+\mathrm{FYM} @ 0$ t/ha, $\mathrm{T}_{5}=0.3 \mathrm{LR}+\mathrm{FYM} @ 2.5 \mathrm{t} / \mathrm{ha}, \mathrm{T}_{6}=0.3 \mathrm{LR}$ $+\mathrm{FYM} @ 5 \mathrm{t} / \mathrm{ha}, \mathrm{T}_{7}=0.5 \mathrm{LR}+\mathrm{FYM} @ 0 \mathrm{t} / \mathrm{ha}$, $\mathrm{T}_{8}=0.5 \mathrm{LR}+\mathrm{FYM} @ 2.5 \mathrm{t} / \mathrm{ha}, \mathrm{T}_{9}=0.5 \mathrm{LR}$ $+F Y M @ 5 t / h a$.$) . The source of lime was$ paper mill sludge having $68 \% \quad \mathrm{CaCO}_{3}$ equivalent. Constant dose of $\mathrm{N}, \mathrm{P}_{2} \mathrm{O}_{5}$ and $\mathrm{K}_{2} \mathrm{O}$ were applied @ 80,40,40 kg/ha, through urea, DAP and muriate of potash, respectively. Required quantities of PMS and FYM were applied during land preparation. After 4 days, mint suckers were planted. Disease free healthy suckers of mint were cut into pieces of 7-10 cm lengths and planted to a depth of 5 to $7 \mathrm{~cm}$ with a row to row spacing of $35 \mathrm{~cm}$. One third of $\mathrm{N}$ and full dose of $\mathrm{P}$ and $\mathrm{K}$ were applied after10 days of planting with full establishment of suckers. Rest 2/3rd of $\mathrm{N}$ was applied of 22 days of planting after the 1 st weeding. Prior to conducting the experiment, were collected after 120 and 180 days of planting and were analysed.

\section{Results and Discussion}

Plant height at 120 day was the lowest $(54 \mathrm{~cm})$ in control (zero lime and zero FYM). It increased significantly with application of lime and FYM. The highest plant height of $82.2 \mathrm{~cm}$ was recorded in the treatment receiving lime@0.3LR and FYM@5ton/ha. At a given level of LR, plant height increased with increasing levels of FYM added and at a given levels of FYM, it increased with increasing levels of lime added.

Plant height at 180 day was the effect lime, FYM and their interactions had significant effects on the plant height. The highest plant height recorded in T-9 (0.5LR + FYM@5t/ha) was statistically at par with T-6 (0.3LR + FYM@5t/ha) but significantly superior to all other treatments. Chlorophyll content at 120 day was the lowest of $0.93 \mathrm{mg} / \mathrm{g}$ leaf in control and increased significantly due to application of lime with the highest value of $1.57 \mathrm{mg} / \mathrm{g}$ in T-6 (0.3LR + FYM @ 5t/ha). 
Table.1 Effect of lime and FYM on plant height in M. arvensis at 120days of planting

\begin{tabular}{|l|l|l|l|l|}
\hline $\begin{array}{l}\text { Level of } \\
\text { Lime }\end{array}$ & \multicolumn{4}{|l|}{ Plant height(cm) } \\
\cline { 2 - 5 } & OFYM & FYM@ 2.5t/ha & FYM@ 5t/ha & MEAN \\
\hline OLR & & & & \\
\hline 0.3 LR & 53.97 & 61.17 & 68.85 & 61.66 \\
\hline 0.5LR & 61.75 & 64.83 & 82.22 & 69.6 \\
\hline MEAN & 69.1 & 70.1 & 79.83 & 73.01 \\
\hline CD(P=0.05) & 61.61 & 65.37 & 77.73 & 68.09 \\
\hline S.E.(m) \pm & LR=3.38** & FYM=3.38** & LRxFYM=NS & \\
\hline CV $(\%)$ & 1.12 & 1.12 & 1.95 & \\
\hline
\end{tabular}

Table.2 Effect of lime and FYM on stem fresh weight and stem dry weight of M. arvensis at 120 days of planting

\begin{tabular}{|c|c|c|c|c|c|c|c|c|}
\hline \multirow{2}{*}{ Lime } & \multicolumn{4}{|c|}{ Stem fresh weight (g/plant) } & \multicolumn{4}{|c|}{ Stem dry weight (g/plant) } \\
\hline & OFYM & $\begin{array}{l}\text { FYM@ } \\
2.5 \mathrm{t} / \mathrm{ha}\end{array}$ & FYM@5t/ha & MEAN & OFYM & $\begin{array}{l}\text { FYM@ } \\
2.5 t / h a\end{array}$ & $\begin{array}{l}\text { FYM@ } \\
5 \text { t/ha }\end{array}$ & MEAN \\
\hline OLR & 64.45 & 70.56 & 98.34 & 77.78 & 19.51 & 21.42 & 27.35 & 22.76 \\
\hline $0.3 \mathrm{LR}$ & 94.09 & 95.27 & 137.23 & $\begin{array}{l}108.8 \\
6 \\
\end{array}$ & 25.69 & 27.63 & 52.3 & 35.21 \\
\hline $0.5 \mathrm{LR}$ & 102.57 & 133.22 & 135.55 & $\begin{array}{l}123.7 \\
8\end{array}$ & 34.66 & 43.22 & 50.89 & 42.93 \\
\hline MEAN & 87.04 & 99.68 & 123.71 & $\begin{array}{l}103.4 \\
8 \\
\end{array}$ & 26.62 & 30.76 & 43.52 & 33.63 \\
\hline $\begin{array}{l}\mathrm{CD} \\
(\mathrm{P}=0.05)\end{array}$ & $\begin{array}{l}\mathrm{LR}=5 . \\
01 * *\end{array}$ & $\begin{array}{l}\text { FYM=3.68* } \\
*\end{array}$ & $\begin{array}{l}\text { LRxFYM=8. } \\
68^{* *}\end{array}$ & & $\begin{array}{l}\mathrm{LR}=3 . \\
68^{* *}\end{array}$ & $\begin{array}{l}\mathrm{FYM}=3.68 \\
* *\end{array}$ & $\begin{array}{l}\text { LRxFYM= } \\
3.68^{* *}\end{array}$ & \\
\hline S.E. $(\mathrm{m}) \pm$ & 1.67 & 1.67 & 2.89 & & 1.23 & 1.23 & 2.13 & \\
\hline $\mathrm{CV}(\%)$ & 4.84 & & & & & 10.95 & & \\
\hline
\end{tabular}

Table.3 Effect of lime and FYM on leaf fresh weight and leaf dry weight of $M$. arvensis at 120 days of planting

\begin{tabular}{|c|c|c|c|c|c|c|c|c|}
\hline \multirow{2}{*}{ Lime } & \multicolumn{4}{|c|}{ leaf fresh weight (g/plant) } & \multicolumn{4}{|c|}{ Leaf dry weight (g/plant) } \\
\hline & OFYM & $\begin{array}{l}\text { FYM@ } \\
2.5 \mathrm{t} / \mathrm{ha}\end{array}$ & FYM@5t/ha & MEAN & OFYM & $\begin{array}{l}\text { FYM@ } \\
2.5 \mathrm{t} / \mathrm{ha}\end{array}$ & $\begin{array}{l}\text { FYM@ } \\
\text { 5t/ha }\end{array}$ & MEAN \\
\hline OLR & 31.17 & 37.65 & 53.69 & 40.83 & 11.60 & 15.71 & 22.68 & 16.66 \\
\hline $0.3 \mathrm{LR}$ & 47.69 & 51.43 & 84.21 & 61.11 & 16.08 & 16.9 & 25.28 & 19.42 \\
\hline $0.5 \mathrm{LR}$ & 43.44 & 64.78 & 73.33 & 60.52 & 16.50 & 21.18 & 25.12 & 20.93 \\
\hline MEAN & 40.76 & 51.29 & 70.41 & 54.51 & 14.73 & 17.93 & 24.36 & 19.00 \\
\hline $\begin{array}{l}\mathrm{CD} \\
(\mathrm{P}=0.05)\end{array}$ & $\begin{array}{l}\mathrm{LR}=5 . \\
44^{* *}\end{array}$ & $\begin{array}{l}\mathrm{FYM}=5.44 * \\
*\end{array}$ & $\begin{array}{l}\text { LRxFYM=9. } \\
43^{* *}\end{array}$ & & $\begin{array}{l}\mathrm{LR}=1.8 \\
0^{* *}\end{array}$ & $\begin{array}{l}\mathrm{FYM}=1.8 \\
0^{* *}\end{array}$ & $\begin{array}{l}\text { LRxFYM= } \\
\text { NS }\end{array}$ & \\
\hline S.E. $(\mathrm{m}) \pm$ & 1.81 & 1.81 & 3.14 & & 0.60 & 0.60 & 1.04 & \\
\hline $\mathrm{CV}(\%)$ & 10.05 & & & & & 9.48 & & \\
\hline
\end{tabular}


Table.4 Effect of lime and FYM on root fresh weight and root dry weight of $M$. arvensis at 120 days of planting

\begin{tabular}{|c|c|c|c|c|c|c|c|c|}
\hline \multirow{2}{*}{$\begin{array}{l}\text { Level of } \\
\text { Lime }\end{array}$} & \multicolumn{4}{|c|}{ Root fresh weight (g/plant) } & \multicolumn{4}{|c|}{ Root dry weight (g/plant) } \\
\hline & OFYM & $\begin{array}{l}\text { FYM@ } \\
2.5 \mathrm{t} / \mathrm{ha}\end{array}$ & FYM@5t/ha & MEAN & OFYM & $\begin{array}{l}\text { FYM@ } \\
2.5 \mathrm{t} / \mathrm{ha}\end{array}$ & $\begin{array}{l}\text { FYM@ } \\
5 \mathrm{t} / \mathrm{ha}\end{array}$ & MEAN \\
\hline OLR & 45.74 & 53.52 & 82.5 & 60.59 & 15.73 & 20.63 & 28.39 & 21.58 \\
\hline $0.3 \mathrm{LR}$ & 76.48 & 98.56 & 146.05 & 107.03 & 28.09 & 37.72 & 49.71 & 38.51 \\
\hline $0.5 \mathrm{LR}$ & 94.50 & 129.71 & 143.57 & 122.59 & 42.97 & 44.26 & 47.26 & 44.83 \\
\hline MEAN & 72.24 & 93.93 & 124.04 & 96.74 & 28.93 & 34.21 & 41.79 & 34.97 \\
\hline $\mathrm{CD}(\mathrm{P}=0.05)$ & $\begin{array}{l}\mathrm{LR}=5.1 \\
6 * *\end{array}$ & $\mathrm{FYM}=5.16^{* *}$ & $\begin{array}{l}\mathrm{LRxFYM}=8.93 \\
* *\end{array}$ & & $\begin{array}{l}\mathrm{LR}=3 . \\
86 * *\end{array}$ & $\mathrm{FYM}=3.86 * *$ & $\begin{array}{l}\text { LRxFYM=6 } \\
.69 * *\end{array}$ & \\
\hline S.E. $(\mathrm{m}) \pm$ & 1.72 & 1.72 & 2.98 & & 1.82 & 1.82 & 2.23 & \\
\hline $\mathrm{CV}(\%)$ & 5.34 & & & & & 11.06 & & \\
\hline
\end{tabular}

Table.5 Effect of lime and FYM on chlorophyll content in the leaves of M. arvensis at 120 days of planting

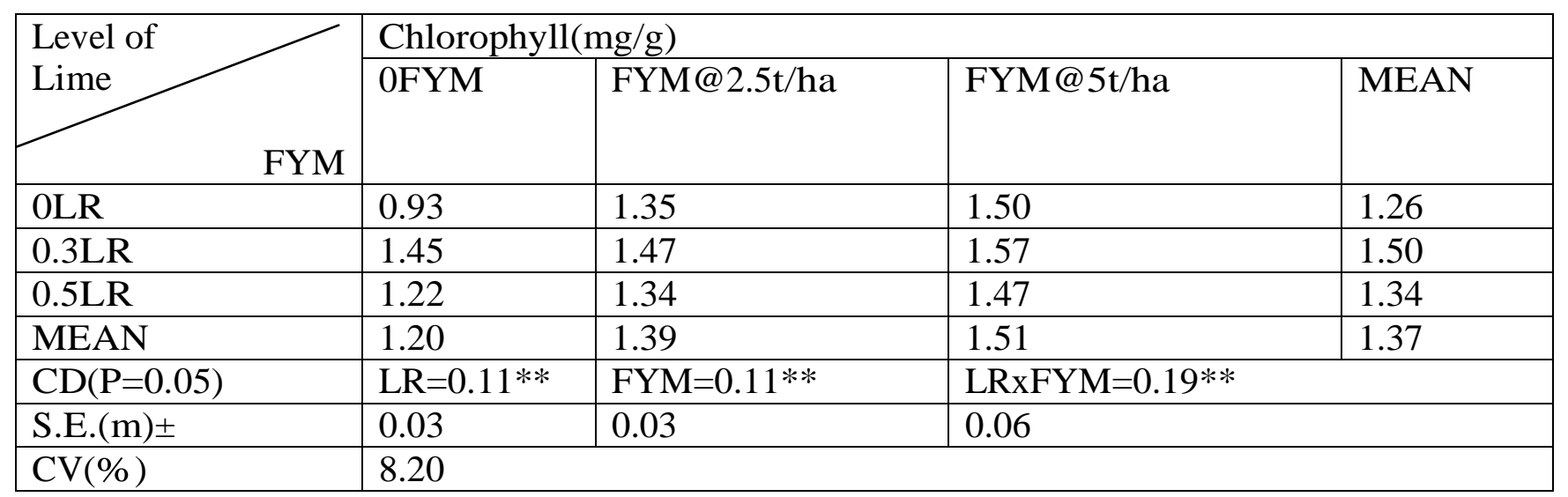

Table.6 Effect of lime and FYM on Leaf yield and Herbage yield of M. arvensis at 120 days of planting

\begin{tabular}{|c|c|c|c|c|c|c|c|c|}
\hline \multirow{2}{*}{ Lime } & \multicolumn{4}{|c|}{ Leaf yield $(\mathrm{kg} / \mathrm{ha})$} & \multicolumn{4}{|c|}{ Herbage yield (kg/ha) } \\
\hline & OFYM & $\begin{array}{l}\text { FYM@ } \\
2.5 \mathrm{t} / \mathrm{ha}\end{array}$ & FYM@5t/ha & MEAN & OFYM & $\begin{array}{l}\text { FYM@ } \\
2.5 \mathrm{t} / \mathrm{ha}\end{array}$ & $\begin{array}{l}\text { FYM@ } \\
5 \text { t/ha }\end{array}$ & MEAN \\
\hline OLR & 4644.44 & 5537.90 & 8031.16 & 6071.17 & $\begin{array}{l}14261 . \\
96\end{array}$ & 16011.83 & 22723.18 & $\begin{array}{l}17665.6 \\
6\end{array}$ \\
\hline $0.3 \mathrm{LR}$ & 7274.80 & 7654.09 & 11918.59 & 8949.16 & $\begin{array}{l}21454 . \\
54\end{array}$ & 21850.84 & 31827.86 & $\begin{array}{l}25044.4 \\
1\end{array}$ \\
\hline $0.5 \mathrm{LR}$ & 6447.49 & 9498.33 & 10843.83 & 8930.22 & $\begin{array}{l}21758 . \\
74\end{array}$ & 29282.37 & 30938.73 & $\begin{array}{l}27326.6 \\
1\end{array}$ \\
\hline MEAN & 6447.50 & 7563.77 & 10264.53 & 7983.52 & $\begin{array}{l}19158 . \\
41\end{array}$ & 22381.68 & 28496.59 & $\begin{array}{l}23345.5 \\
6 \\
\end{array}$ \\
\hline $\mathrm{CD}(\mathrm{P}=0.05)$ & $\begin{array}{l}\mathrm{LR}=63 \\
1.51 * *\end{array}$ & $\begin{array}{l}\mathrm{FYM}=631.51 \\
* *\end{array}$ & $\begin{array}{l}\text { LRxFYM=109 } \\
3.81 * *\end{array}$ & & $\begin{array}{l}\mathrm{LR}=91 \\
0.70 * *\end{array}$ & $\begin{array}{l}\mathrm{FYM}=910.70 \\
* *\end{array}$ & $\begin{array}{l}\text { LRxFYM=1 } \\
577.37 * *\end{array}$ & \\
\hline S.E.(m) \pm & 210.64 & 210.64 & 364.85 & & 303.76 & 303.76 & 526.14 & \\
\hline $\mathrm{CV}(\%)$ & 7.92 & & & & & 3.90 & & \\
\hline
\end{tabular}


Table.7 Effect of lime and FYM on Oil content and oil yield of M. arvensis at 120 days of planting

\begin{tabular}{|c|c|c|c|c|c|c|c|c|}
\hline \multirow{2}{*}{$\begin{array}{l}\text { Level of } \\
\text { Lime }\end{array}$} & \multicolumn{4}{|c|}{$\%$ Oil content } & \multicolumn{4}{|c|}{ Oil yield $(\mathrm{kg} / \mathrm{ha})$} \\
\hline & OFYM & $\begin{array}{l}\text { FYM@ } \\
2.5 \mathrm{t} / \mathrm{ha}\end{array}$ & FYM@5t/ha & MEAN & OFYM & $\begin{array}{l}\text { FYM@ } \\
2.5 \mathrm{t} / \mathrm{ha}\end{array}$ & $\begin{array}{l}\text { FYM@ } \\
5 \text { t/ha }\end{array}$ & MEAN \\
\hline OLR & 0.13 & 0.27 & 0.37 & 0.26 & 7.52 & 18.32 & 29.61 & 18.48 \\
\hline $0.3 \mathrm{LR}$ & 0.27 & 0.3 & 0.4 & 0.32 & 14.55 & 20.57 & 43.98 & 26.36 \\
\hline $0.5 \mathrm{LR}$ & 0.27 & 0.23 & 0.33 & 0.28 & 12.89 & 31.25 & 39.37 & 27.84 \\
\hline MEAN & 0.22 & 0.27 & 0.37 & 0.29 & 11.65 & 23.38 & 37.65 & 24.23 \\
\hline $\mathrm{CD}(\mathrm{P}=0.05)$ & $\mathrm{LR}=\mathrm{NS}$ & $\mathrm{FYM}=008 * *$ & LRxFYM=NS & & $\begin{array}{l}\mathrm{LR}=5.0 \\
0 * *\end{array}$ & $\begin{array}{l}\mathrm{FYM}=5.00 * \\
*\end{array}$ & $\begin{array}{l}\text { LRxFYM= } \\
\text { NS }\end{array}$ & \\
\hline S.E. $(\mathrm{m}) \pm$ & 0.02 & 0.02 & 0.04 & & 1.67 & 1.67 & 2.89 & \\
\hline $\mathrm{CV}(\%)$ & 28.53 & & & & & 20 & & \\
\hline
\end{tabular}

Table.8 Effect of lime and FYM on plant height in M. arvensis at 180 days of planting

\begin{tabular}{|c|c|c|c|c|}
\hline Level of & Plant height & & & \\
\hline Lime & OFYM & FYM@2.5t/ha & FYM@5t/ha & MEAN \\
\hline & & & & \\
\hline OLR & 50.97 & 51.9 & 61.1 & 54.66 \\
\hline $0.3 \mathrm{LR}$ & 56.02 & 64.95 & 77.07 & 66.01 \\
\hline $0.5 \mathrm{LR}$ & 69.7 & 73.25 & 80.2 & 74.38 \\
\hline MEAN & 58.90 & 63.37 & 73.79 & 65.06 \\
\hline $\mathrm{CD}(\mathrm{P}=0.05)$ & $\mathrm{LR}=3.09^{* *}$ & $\mathrm{FYM}=3.09 * *$ & LRxFYM=5.36 & \\
\hline S.E. $(\mathrm{m}) \pm$ & 1.03 & 1.03 & 1.78 & \\
\hline $\mathrm{CV}(\%)$ & 4.76 & & & \\
\hline
\end{tabular}

Table.9 Effect of lime and FYM on stem fresh weight and stem dry weight of M. arvensis at 180 days of planting

\begin{tabular}{|c|c|c|c|c|c|c|c|c|}
\hline \multirow{3}{*}{\begin{tabular}{|l|} 
Level of \\
Lime \\
FYM
\end{tabular}} & \multicolumn{4}{|c|}{ Stem fresh weight (g/plant) } & \multicolumn{4}{|c|}{ Stem dry weight (g/plant) } \\
\hline & OFYM & $\begin{array}{l}\text { FYM@ } \\
2.5 \mathrm{t} / \mathrm{ha}\end{array}$ & FYM@5t/ha & MEAN & OFYM & $\begin{array}{l}\text { FYM@ } \\
2.5 \mathrm{t} / \mathrm{ha}\end{array}$ & $\begin{array}{l}\text { FYM@ } \\
5 \text { t/ha }\end{array}$ & MEAN \\
\hline & 55.16 & 64.07 & 73.41 & 64.21 & 20.44 & 23.37 & 30.34 & 24.72 \\
\hline $0.3 \mathrm{LR}$ & 88.42 & 96.14 & 132.29 & $\begin{array}{l}105.6 \\
2\end{array}$ & 29.73 & 31.56 & 44.67 & 35.32 \\
\hline $0.5 \mathrm{LR}$ & 123.87 & 133.81 & 164.67 & $\begin{array}{l}140.7 \\
4\end{array}$ & 32.82 & 44.24 & 56.63 & 44.56 \\
\hline MEAN & 89.09 & 98.01 & 123.45 & $\begin{array}{l}103.5 \\
2\end{array}$ & 27.66 & 33.06 & 43.88 & 34.87 \\
\hline $\mathrm{CD}(\mathrm{P}=0.05)$ & $\begin{array}{l}\mathrm{LR}=5.4 \\
6 * *\end{array}$ & $\mathrm{FYM}=5.46^{*}$ & $\begin{array}{l}\text { LR } x \\
\text { FYM }=9.46^{* *}\end{array}$ & & $\begin{array}{l}\mathrm{LR}=4 . \\
44^{* *}\end{array}$ & $\begin{array}{l}\mathrm{FYM}=4.44 * \\
*\end{array}$ & $\begin{array}{l}\mathrm{LR} \\
\mathrm{xFYM}=\mathrm{NS}\end{array}$ & \\
\hline S.E. $(\mathrm{m}) \pm$ & 2.58 & 2.58 & 3.16 & & 1.48 & 1.48 & 2.57 & \\
\hline $\mathrm{CV}(\%)$ & 5.28 & & & & & 12.74 & & \\
\hline
\end{tabular}


Table.10 Effect of lime and FYM on leaf fresh weight and leaf dry weight of M. arvensis at 180 days of planting

\begin{tabular}{|c|c|c|c|c|c|c|c|c|}
\hline \multirow{2}{*}{ Lime } & \multicolumn{4}{|c|}{ leaf fresh weight (g/plant) } & \multicolumn{4}{|c|}{ Leaf dry weight (g/plant) } \\
\hline & OFYM & $\begin{array}{l}\text { FYM@ } \\
2.5 \mathrm{t} / \mathrm{ha}\end{array}$ & FYM@5t/ha & MEAN & OFYM & $\begin{array}{l}\text { FYM@ } \\
2.5 \mathrm{t} / \mathrm{ha}\end{array}$ & $\begin{array}{l}\text { FYM@ } \\
\text { 5t/ha }\end{array}$ & MEAN \\
\hline OLR & 24.66 & 29.66 & 37.34 & 30.55 & 7.29 & 8.42 & 9.47 & 8.39 \\
\hline $0.3 \mathrm{LR}$ & 31.33 & 38.18 & 43.06 & 37.52 & 9.45 & 11.11 & 14.51 & 11.69 \\
\hline $0.5 \mathrm{LR}$ & 36.51 & 43.45 & 56.29 & 45.41 & 11.32 & 14.19 & 19.06 & 14.85 \\
\hline MEAN & 30.83 & 37.1 & 45.56 & 37.80 & 9.35 & 11.24 & 14.34 & 11.67 \\
\hline $\begin{array}{l}\text { CD } \\
(P=0.05)\end{array}$ & $\begin{array}{l}\mathrm{LR}=3 . \\
67 * *\end{array}$ & $\begin{array}{l}\mathrm{FYM}=3.67 * \\
*\end{array}$ & $\begin{array}{l}\text { LRxFYM=N } \\
S\end{array}$ & & $\begin{array}{l}\mathrm{LR}=0 . \\
88^{* *}\end{array}$ & $\begin{array}{l}\mathrm{FYM}=0.88 \\
* *\end{array}$ & $\begin{array}{l}\text { LRxFYM= } \\
1.52 * *\end{array}$ & \\
\hline S.E. $(\mathrm{m}) \pm$ & 1.22 & 1.22 & 2.11 & & 0.29 & 0.29 & 0.51 & \\
\hline $\mathrm{CV}(\%)$ & 9.71 & & & & & 7.5 & & \\
\hline
\end{tabular}

Table.11 Effect of lime and FYM on root fresh weight and root dry weight of $M$. arvensis at 180 days of planting

\begin{tabular}{|c|c|c|c|c|c|c|c|c|}
\hline \multirow{3}{*}{$\underbrace{\text { Level of }}_{\text {FYM }}$} & \multicolumn{4}{|c|}{ Root fresh weight (g/plant) } & \multicolumn{4}{|c|}{ Root dry weight (g/plant) } \\
\hline & OFYM & $\begin{array}{l}\text { FYM@ } \\
2.5 \mathrm{t} / \mathrm{ha}\end{array}$ & FYM@5t/ha & MEAN & OFYM & $\begin{array}{l}\text { FYM@ } \\
2.5 \mathrm{t} / \mathrm{ha}\end{array}$ & $\begin{array}{l}\text { FYM@ } \\
\text { 5t/ha }\end{array}$ & MEAN \\
\hline & & & & & & & & \\
\hline OLR & 62.25 & 71.12 & 86.25 & 73.21 & 22.32 & 30.83 & 23.28 & 25.48 \\
\hline $0.3 \mathrm{LR}$ & 85.85 & 99.19 & 104.27 & 96.44 & 35.85 & 46.83 & 59.9 & 47.53 \\
\hline $0.5 \mathrm{LR}$ & 135.71 & 157.21 & 152 & 148.31 & 54.17 & 62.75 & 62.32 & 59.75 \\
\hline MEAN & 94.60 & 109.17 & 114.17 & 109.84 & 37.45 & 46.80 & 48.5 & 45.36 \\
\hline $\begin{array}{l}\mathrm{CD} \\
(\mathrm{P}=0.05)\end{array}$ & $\begin{array}{l}\mathrm{LR}=3 . \\
57 * *\end{array}$ & $\begin{array}{l}\mathrm{FYM}=3.57 \\
* *\end{array}$ & $\begin{array}{l}\text { LRxFYM=6 } \\
.18^{* *}\end{array}$ & & $\begin{array}{l}\mathrm{LR}=3 . \\
21 * *\end{array}$ & $\begin{array}{l}\mathrm{FYM}=3.21 \\
* *\end{array}$ & $\begin{array}{l}\text { LRxFYM } \\
=5.56^{* * *}\end{array}$ & \\
\hline S.E. $(\mathrm{m}) \pm$ & 1.19 & 1.19 & 2.06 & & 1.07 & 1.07 & 1.86 & \\
\hline $\mathrm{CV}(\%)$ & 3.25 & & & & & 7.08 & & \\
\hline
\end{tabular}

Table.12 Effect of lime and FYM on chlorophyll content in the leaves of M. arvensis at 180 days of planting

\begin{tabular}{|c|c|c|c|c|}
\hline Level of & Chlorophyll & ig/g) & & \\
\hline Lime & OFYM & FYM@2.5t/ha & FYM@5t/ha & MEAN \\
\hline & & & & \\
\hline OLR & 0.7 & 1.16 & 1.47 & 1.11 \\
\hline $0.3 \mathrm{LR}$ & 1.41 & 1.36 & 1.58 & 1.45 \\
\hline $0.5 \mathrm{LR}$ & 1.38 & 1.22 & 1.51 & 1.37 \\
\hline MEAN & 1.17 & 1.25 & 1.52 & 1.31 \\
\hline $\mathrm{CD}(\mathrm{P}=0.05)$ & $\mathrm{LR}=0.081 * *$ & $\mathrm{FYM}=0.081 * *$ & $\mathrm{LRxFYM}=0.141 * *$ & \\
\hline S.E. $(\mathrm{m}) \pm$ & 0.02 & 0.02 & 0.04 & \\
\hline $\mathrm{CV}(\%)$ & 6.23 & & & \\
\hline
\end{tabular}


Table.13 Effect of lime and FYM on Leaf yield and Herbage yield of M. arvensis at 180 days of planting

\begin{tabular}{|c|c|c|c|c|c|c|c|c|}
\hline \multirow{2}{*}{$\begin{array}{l}\text { Level of } \\
\text { Lime }\end{array}$} & \multicolumn{4}{|c|}{ Leaf yield (kg/ha) } & \multicolumn{4}{|c|}{ Herbage yield $(\mathrm{kg} / \mathrm{ha})$} \\
\hline & OFYM & $\begin{array}{l}\text { FYM@ } \\
2.5 \mathrm{t} / \mathrm{ha}\end{array}$ & $\begin{array}{l}\text { FYM@5t } \\
\text { /ha }\end{array}$ & MEAN & OFYM & $\begin{array}{l}\text { FYM@ } \\
2.5 t / h a\end{array}$ & $\begin{array}{l}\text { FYM@ } \\
5 t / h a\end{array}$ & MEAN \\
\hline OLR & $\begin{array}{l}3779 . \\
81\end{array}$ & 4447.22 & 5485.50 & $\begin{array}{l}4570.8 \\
4\end{array}$ & $\begin{array}{l}12133.6 \\
3\end{array}$ & 14051.1 & 16363.69 & $\begin{array}{l}14182 \\
.81\end{array}$ \\
\hline $0.3 \mathrm{LR}$ & $\begin{array}{l}4732 . \\
60\end{array}$ & 5569.61 & 623 & $\begin{array}{l}5513.2 \\
4\end{array}$ & $\begin{array}{l}17910.1 \\
1\end{array}$ & 19850.10 & 26063.68 & $\begin{array}{l}21274 \\
.63\end{array}$ \\
\hline $0.5 \mathrm{LR}$ & $\begin{array}{l}5309 . \\
67\end{array}$ & 6499.41 & 8415.30 & $\begin{array}{l}6741.4 \\
5\end{array}$ & $\begin{array}{l}23699.2 \\
4\end{array}$ & 26554.05 & 33013.05 & $\begin{array}{l}27755 \\
.45\end{array}$ \\
\hline MEAN & $\begin{array}{l}4607 . \\
36\end{array}$ & 5505.41 & 6712.76 & $\begin{array}{l}5608.5 \\
1\end{array}$ & $\begin{array}{l}17914.3 \\
3\end{array}$ & 20151.75 & 25146.81 & $\begin{array}{l}21070 \\
.96\end{array}$ \\
\hline $\begin{array}{l}C D \\
(P=0.05)\end{array}$ & $\begin{array}{l}\mathrm{LR}=4 \\
95.32 * \\
*\end{array}$ & $\begin{array}{l}\mathrm{FYM}=49 \\
5.32 * *\end{array}$ & $\begin{array}{l}\text { LRxFYM } \\
=\mathrm{NS}\end{array}$ & & $\begin{array}{l}\mathrm{LR}=202 \\
2.64 * *\end{array}$ & $\begin{array}{l}\mathrm{FYM}=202 \\
2.64 * *\end{array}$ & $\begin{array}{l}\text { LRxFYM } \\
=\mathrm{NS}\end{array}$ & \\
\hline S.E. $(\mathrm{m}) \pm$ & 1.18 & 1.18 & 2.04 & & 674.67 & 674.67 & 1168.54 & \\
\hline $\mathrm{CV}(\%)$ & 8.84 & & & & & 9.61 & & \\
\hline
\end{tabular}

Table.14 Effect of lime and FYM on Oil content and oil yield of M. arvensis at 180 days of planting

\begin{tabular}{|c|c|c|c|c|c|c|c|c|}
\hline \multirow{2}{*}{$\begin{array}{l}\text { Level of } \\
\text { Lime }\end{array}$} & \multicolumn{4}{|c|}{$\%$ Oil content } & \multicolumn{4}{|c|}{ Oil yield(kg/ha) } \\
\hline & OFYM & $\begin{array}{l}\text { FYM@ } \\
2.5 \mathrm{t} / \mathrm{ha}\end{array}$ & FYM@5t/ha & MEAN & OFYM & $\begin{array}{l}\text { FYM@ } \\
2.5 \mathrm{t} / \mathrm{ha}\end{array}$ & $\begin{array}{l}\text { FYM@ } \\
5 t / h a\end{array}$ & MEAN \\
\hline OLR & 0.167 & 0.333 & 0.367 & 0.289 & 5.93 & 14.76 & 20.25 & 13.65 \\
\hline $0.3 \mathrm{LR}$ & 0.2 & 0.267 & 0.367 & 0.278 & 9.47 & 15.11 & 23.14 & 15.90 \\
\hline $0.5 \mathrm{LR}$ & 0.2 & 0.333 & 0.4 & 0.311 & 10.62 & 21.48 & 30.17 & 20.75 \\
\hline MEAN & 0.189 & 0.311 & 0.378 & 0.293 & 8.67 & 17.12 & 24.52 & 16.76 \\
\hline $\begin{array}{l}\text { CD } \\
(P=0.05)\end{array}$ & $\begin{array}{l}\mathrm{LR}=\mathrm{N} \\
\mathrm{S}\end{array}$ & $\begin{array}{l}\mathrm{FYM}=0.05 \\
0 * *\end{array}$ & $\begin{array}{l}\text { LRxFYM=N } \\
S\end{array}$ & & $\begin{array}{l}\mathrm{LR}=3 \\
.53 * *\end{array}$ & $\begin{array}{l}\mathrm{FYM}=3.53 \\
* *\end{array}$ & $\begin{array}{l}\text { LRxFYM } \\
=\mathrm{NS}\end{array}$ & \\
\hline S.E. $(\mathrm{m}) \pm$ & 0.02 & 0.02 & 0.03 & & 1.18 & 1.18 & 2.04 & \\
\hline $\mathrm{CV}(\%)$ & 16.93 & & & & & 21.07 & & \\
\hline
\end{tabular}


Table.15 Correlation coefficient values among different plant parameters at 120 days of planting

\begin{tabular}{|c|c|c|c|c|c|c|c|c|c|c|c|c|}
\hline & $\begin{array}{l}\text { PLANT } \\
\text { HEIGHT }\end{array}$ & \begin{tabular}{|l} 
STEM \\
FRESH \\
WEIGHT
\end{tabular} & $\begin{array}{l}\text { STEM } \\
\text { DRY } \\
\text { WEIGHT }\end{array}$ & $\begin{array}{l}\text { LEAF } \\
\text { FRESH } \\
\text { WEIGHT }\end{array}$ & $\begin{array}{l}\text { LEAF } \\
\text { DRY } \\
\text { WEIGHT }\end{array}$ & $\begin{array}{l}\text { ROOT } \\
\text { FRESH } \\
\text { WEIGHT }\end{array}$ & \begin{tabular}{|l|} 
ROOT \\
DRY \\
WEIGHT
\end{tabular} & $\begin{array}{l}\text { LEAF } \\
\text { YIELD }\end{array}$ & $\begin{array}{l}\text { HERB AGE } \\
\text { YIELD }\end{array}$ & $\begin{array}{l}\% \text { OIL } \\
\text { CONTENT }\end{array}$ & $\begin{array}{l}\text { OIL } \\
\text { YIELD }\end{array}$ & $\begin{array}{l}\text { CHLOROPH } \\
\text { YLL }\end{array}$ \\
\hline $\begin{array}{l}\text { PLANT } \\
\text { HEIGHT }\end{array}$ & & $0.91 * *$ & $0.93 * *$ & $0.93 * *$ & $0.94 * *$ & $0.92 * *$ & $0.88 * *$ & $0.93 * *$ & $0.93 * *$ & $0.81 * *$ & $0.91 * *$ & $0.69^{*}$ \\
\hline $\begin{array}{l}\text { STEMFRESH } \\
\text { WEIGHT }\end{array}$ & & & $0.96^{* *}$ & $0.94 * *$ & $0.88 * *$ & $0.98 * *$ & $0.93 * *$ & $0.95 * *$ & $0.99 * *$ & 0.59 & $0.86 * *$ & 0.60 \\
\hline $\begin{array}{l}\text { STEMDRY } \\
\text { WEIGHT }\end{array}$ & & & & $0.93 * *$ & $0.85 * *$ & $0.97 * *$ & $0.92 * *$ & $0.93 * *$ & $0.96 * *$ & 0.56 & $0.86 * *$ & 0.50 \\
\hline $\begin{array}{l}\text { LEAFFRESH } \\
\text { WEIGHT }\end{array}$ & & & & & $0.93 * *$ & $0.95 * *$ & $0.84 * *$ & $1.00 * *$ & $0.97 * *$ & 0.73 & $0.95 * *$ & $0.71 *$ \\
\hline $\begin{array}{l}\text { LEAFDRY } \\
\text { WEIGHT. }\end{array}$ & & & & & & $0.86 * *$ & $0.75^{*}$ & $0.93 * *$ & $0.91 * *$ & $0.83 * *$ & $0.97 * *$ & $0.75^{*}$ \\
\hline $\begin{array}{l}\text { ROOTFRESH } \\
\text { WEIGHT }\end{array}$ & & & & & & & $0.96 * *$ & $0.95 * *$ & $0.98 * *$ & 0.61 & $0.86^{* *}$ & 0.60 \\
\hline $\begin{array}{l}\text { ROOTDRY } \\
\text { WEIGHT }\end{array}$ & & & & & & & & $0.84 * *$ & $0.91 * *$ & 0.58 & $0.72 *$ & 0.55 \\
\hline LEAFYIELD & & & & & & & & & $0.97 * *$ & $0.73^{*}$ & $0.95 * *$ & $0.71 *$ \\
\hline $\begin{array}{l}\text { HERBAGE } \\
\text { YIELD }\end{array}$ & & & & & & & & & & 0.65 & $0.90 * *$ & 0.65 \\
\hline $\begin{array}{l}\text { \%OILCONTE } \\
\text { NT }\end{array}$ & & & & & & & & & & & $0.77 *$ & $0.90 * *$ \\
\hline OILYIELD & & & & & & & & & & & & $0.73 *$ \\
\hline CHLOROPHY & & & & & & & & & & & & \\
\hline
\end{tabular}

$\mathrm{n}=9, \mathrm{df}=7, \mathrm{r}=0.67$ at $5 \%$ level, $\mathrm{r}=0.80=$ at 15 level. $*=$ Significant at $5 \%$ level $* *=$ Significant at $5 \%$ level 
Table.16 Correlation coefficient values among different plant parameter 180 days of planting

\begin{tabular}{|c|c|c|c|c|c|c|c|c|c|c|c|c|}
\hline & $\begin{array}{l}\text { PLANT } \\
\text { HEIGHT }\end{array}$ & $\begin{array}{c}\text { STEM } \\
\text { FRESH } \\
\text { WEIGHT }\end{array}$ & $\begin{array}{l}\text { STEMDRY } \\
\text { WEIGHT }\end{array}$ & $\begin{array}{l}\text { LEAFFRES } \\
\text { H WEIGHT }\end{array}$ & $\begin{array}{l}\text { LEAFDRY } \\
\text { WEIGHT }\end{array}$ & $\begin{array}{l}\text { ROOTFRESH } \\
\text { WEIGHT }\end{array}$ & $\begin{array}{l}\text { ROOTDRY } \\
\text { WEIGHT }\end{array}$ & $\begin{array}{l}\text { LEAF } \\
\text { YIELD }\end{array}$ & $\begin{array}{l}\text { HERB AG } \\
\text { EYIELD }\end{array}$ & $\begin{array}{l}\% \text { OIL } \\
\text { CONTENT }\end{array}$ & $\begin{array}{l}\text { OIL } \\
\text { YIELD }\end{array}$ & $\begin{array}{l}\text { CHLORO } \\
\text { PHYLL }\end{array}$ \\
\hline $\begin{array}{l}\text { PLANT } \\
\text { HEIGHT }\end{array}$ & & $0.96 * *$ & $0.94 * *$ & $0.92 * *$ & $0.93 * *$ & $0.96 * *$ & $0.96 * *$ & $0.90 * *$ & $0.96 * *$ & 0.57 & $0.79 *$ & 0.65 \\
\hline $\begin{array}{l}\text { STEMFRESH } \\
\text { WEIGHT }\end{array}$ & & & $0.95 * *$ & $0.91 * *$ & $0.96 * *$ & $0.96 * *$ & $0.96 * *$ & $0.90 * *$ & $1.00 * *$ & 0.47 & $0.72 *$ & 0.61 \\
\hline $\begin{array}{l}\text { STEMDRY } \\
\text { WEIGHT }\end{array}$ & & & & $0.97 * *$ & $0.99 * *$ & $0.90 * *$ & $0.89 * *$ & $0.97 * *$ & $0.97 * *$ & 0.66 & $0.87 * *$ & 0.62 \\
\hline $\begin{array}{l}\text { LEAFFRESH } \\
\text { WEIGHT }\end{array}$ & & & & & $0.97 * *$ & $0.84 * *$ & $0.85^{* *}$ & $1.00 * *$ & $0.94 * *$ & $0.72 *$ & $0.92 * *$ & $0.67 *$ \\
\hline $\begin{array}{l}\text { LEAFDRY } \\
\text { WEIGHT. }\end{array}$ & & & & & & $0.89 * *$ & $0.89 * *$ & $0.97 * *$ & $0.98 * *$ & 0.63 & $0.86 * *$ & 0.59 \\
\hline $\begin{array}{l}\text { ROOTFRESH } \\
\text { WEIGHT }\end{array}$ & & & & & & & $0.98 * *$ & $0.83 * *$ & $0.95 * *$ & 0.46 & 0.68 & 0.55 \\
\hline $\begin{array}{l}\text { ROOTDRY } \\
\text { WEIGHT }\end{array}$ & & & & & & & & $0.84 * *$ & $0.95^{* *}$ & 0.48 & $0.70^{*}$ & 0.62 \\
\hline LEAFYIELD & & & & & & & & & $0.94 * *$ & $0.72 *$ & $0.91 * *$ & 0.63 \\
\hline $\begin{array}{l}\text { HERBAGE } \\
\text { YIELD }\end{array}$ & & & & & & & & & & 0.52 & $0.77 *$ & 0.62 \\
\hline $\begin{array}{l}\% \text { OIL } \\
\text { CONTENT }\end{array}$ & & & & & & & & & & & $0.93 * *$ & 0.56 \\
\hline OIL YIELD & & & & & & & & & & & & 0.62 \\
\hline $\begin{array}{l}\text { CHLOROPH } \\
\text { YL }\end{array}$ & & & & & & & & & & & & \\
\hline
\end{tabular}

$\mathrm{n}=9, \mathrm{df}=7, \mathrm{r}=0.67$ at $5 \%$ level, $\mathrm{r}=0.80=$ at 15 level. $*=$ Significant at $5 \%$ level $* *=$ Significant at $5 \%$ level 
At a given of lime applied chlorophyll content increased with increasing levels of FYM added. At a given level of FYM chlorophyll content increased upto 0.3LR level and decreased at 0.5LR level. Effect of lime and FYM and their interaction on chlorophyll content was significant. The highest chlorophyll contentinT-6 (0.3LR + FYM@5t/ha) was statistically at par with that in T-9 (0.5LR + FYM@5t/ha) but was significantly superior to all other treatments. Chlorophyll content at 180 day was the lowest (0.7 $\mathrm{gm} / \mathrm{g}$ ) in the control and increased significantly with application of lime and FYM. The highest chlorophyll content of $1.58 \mathrm{mg} / \mathrm{g}$ was recorded in T-6 $(0.3 \mathrm{LR}+$ FYM@5 ton/ha). The highest chlorophyll content in T-6 was statistically at par with T-9 $(0.5 \mathrm{LR}+$ FYM@5ton/ha) but significantly superior to other treatments.

Values of all the growth parameters as well as leaf, herbage and oil yields and \% oil content were the highest with application of lime@0.3LR along with FYM@5ton/ha at 120 days of planting. The leaf, herbage and oil yields in this treatment was $119.18 \mathrm{q} / \mathrm{ha}$, $318.27 \mathrm{q} / \mathrm{ha}$ and $43.98 \mathrm{~kg} / \mathrm{ha}$, respectively as against the leaf, herbage and oil yields of $46.44 \mathrm{q} / \mathrm{ha}, \quad 142.61 \mathrm{q} / \mathrm{ha}$ and $7.52 \mathrm{~kg} / \mathrm{ha}$ in control (0LR+0FYM). At 180 days of planting, different growth parameters like plant height, weights of root, stem and leaf/plant, leaf, herbage yield, $\%$ oil content and oil yield were the highest with application of lime@0.5LR along with FYM@5ton/ha. The leaf, herbage and oil yield in this treatment were $84.2 \mathrm{q} / \mathrm{ha}, 33.0 \mathrm{q} / \mathrm{ha}$ and $30.2 \mathrm{~kg} / \mathrm{ha}$, respectively as against the leaf, herbage and oil yields of $37.8 \mathrm{q} / \mathrm{ha}, 121.3 \mathrm{q} / \mathrm{ha}$ and $5.9 \mathrm{~kg} / \mathrm{ha}$, respectively in control. All the growth parameters data recorded on mint plant has been presented in table 1-14.

Significant positive correlation existed among different growth parameters both at 120 and
180 days of planting (Table 15 and 16). Plant height, dry and fresh weights of stems, leaves, leaf and herbage yield and oil yield possessed highly significant positive correlation with the dry and fresh root weights. This indicated that the plant growth in $M$. arvensis was significantly related to the root growth in soil. Dry and fresh weight of leaves, leaf and herbage yields and oil yield were positively correlated with plant height. Oil yield was positively correlated with the leaf and herbage yields. Positive correlation existed between $\%$ oil content and oil yield in Mentha arvensis. Positive relationship of $\%$ oil content with plant height, stem fresh and dry weight, leaf fresh and dry weight was obtained only at 120 days of planting. A highly significant positive correlation was obtained between the chlorophyll content and percent oil content at 120 days of planting.

The leaf, herbage and oil yields in Mentha arvensis increased significantly with application of lime and FYM.

Maximum leaf, herbage and oil yields in the $1^{\text {st }}$ harvesting (i.e. after 120 days of planting) were obtained with application of lime@0.3LR and FYM@5t/ha, whereas these yields at those in the second harvesting (i.e. after 60 days of $1^{\text {st }}$ harvesting) were the maximum when lime was applied @0.5LR with FYM@5.0t/ha. The lowest yields were obtained when no lime and FYM were added. Application of lime had no effect in increasing the oil content in plant, whereas FYM had a significant effect in increasing the oil content in $M$. arvensis.

\section{Acknowledgments}

The author thankfully acknowledges the critical comments and suggestions on the manuscript received from Dr. M.S. Malik (Prof. \& Chairman, SAF) Faculty of Forestry, BAU, Ranchi, Jharkhand. 


\section{References}

Anwar, M., Chand, S. and Patra, D.D. 2010. Effect of graded level of NPKon fresh herb yield, oilyield and oil composition of six cultivars of menthol mint, Indian Journal Natural Product Resources. 1(1):74-79.

Chand,S., Patra, N.K. ,Anwar, M. and Patra D.D.2004. Agronomy and uses of Menthol mint (Mentha arvensis) Indian perspective. Proceeding of the National Science Academy.3:269-297.

Clark, S. M., Horwath, W.R., Shennan, C. and Scow, K.M.1998. Changes in soil chemical properties resulting from organic and low-input farming practices. Agronomy Journal. 90:662671.

Farooqi, A.A. and Sreeramu, B.S.2001.CultivationofMedicinalandaro maticplantcrops.UniversityPressHydera bad, pp410-411.

Kumar, S., Bahl, J.R., Bansal, P.R., Kukreja, A.K., Garg, S.N., Nagvi, A.A., Luthra, R. and Sharma, S. 2002. Profiles of the essential oils of Indian menthol mint (Mentha arvensis) cultivars at different stages of crop growth in northern plain.Journal of Medicinal and
Aromatic Plant Sciences.2(1): 774-786.

Kumar, S., Srivastava, R.K., Singh A.K., Kalra, A., Tomar, V.K.S. and Bansal, R.P. 2001. Higher yields and profit from crop rotations permitting integration of mediculture with agriculture in the Indo-Gangetic plains. Current Sciences. 80(4): 563-566.

Lawrence BM, 1985. A review of the world production of essential oils. 1984. Perfumer andFlavorist.10(5):1-15.

Lorenzi, H. and Matos, F.J.A. 2002. Medicinal plants of Brazil: native and exotic, Plantarum Institute, Nova Odessa. pp 254.

Singh, M.K. and Saini, S.S. 2008. Planting Dates, Mulch and Herbicide Rate Effects on the Growth, Yield and Physicochemical Properties of Menthol mint (Mentha arvensis). Weed Technology. 22(4): 691-698.

Tassou, C. C. Nychas, G.J.E. and Skandamis, P.N. 2004. Herbs and spices and antimicrobials. In: Peter, K.V.ed. Handbook of Herbsand Spices. A bington, Cambridge CB1 6AH, England, Wood head Publishing Limited, Abington Hall.pp.35-53.

\section{How to cite this article:}

Sunita Kumari, P.K. Das, Abhay Kumar and Oraon, P.R. 2017. Effect of Lime and FYM on the Herb Yield of Mint (Mentha arvensis) in an Acid Soil Odisha. Int.J.Curr.Microbiol.App.Sci. 6(11): 3340-3350. doi: https://doi.org/10.20546/ijcmas.2017.611.392 